accepted. 6 of these 8 patients showed full recovery of thyroid function, with a lower levothyroxine dose evident in 5 cases. 2 of these patients had associated co-morbidities Down syndrome and Deletion 1q21.1 syndrome. Overall, 4 patients with $\mathrm{CHD}$ had associated co-morbidities, whereas no other forms of $\mathrm{CH}$ had any.

Conclusion The incidence of $\mathrm{CH}$ in the local area is approximately 1 in 1200, much higher than the UK average of 1 in 4000. Dyshormonogenesis accounts for the greatest proportion of $\mathrm{CH}(40 \%)$ compared to established literature where it is shown to be $10 \%-20 \%$. Further investigation, therefore, is required to see why $\mathrm{CHD}$ is more common in this area. There is insufficient information available to draw conclusions with regards to likelihood of recovery. However, the study is suggestive of recovery being more likely with a lower dose of levothyroxine, and for those patients with associated syndromes. In order to test this hypothesis, a larger study needs to be undertaken.

\section{G237(P) EFFECTIVENESS OF THE SENSOR AUGMENTED PUMP IN MANAGING TYPE I DIABETES MELLITUS}

S Kattera, JM Robinson, JOoi Enn. Child Health, Wrightington, Wigan and Leigh NHS Foundation Trust, Manchester, UK

\subsection{6/archdischild-2018-rcpch.231}

Aim Type I Diabetes Mellitus is a chronic metabolic disorder with an incidence of 24000 children in the UK. NICE has recommended the use of the sensor augmented pump (SAP) in managing short and long term diabetes related complications. Limited literature is available in the paediatric population. SAP is designed to allow continuous glucose monitoring, with realtime adjustment of insulin; making it superior to other pump systems. SAP was assessed in a trial of children; that were previously on insulin pumps with limited success in managing hypoglycaemic episodes. The aim of the audit was to identify how effective SAP was in reducing HBA1c and preventing hypoglycaemic episodes.

Method 14 children were commenced on SAP between 20162017 March. Evidence was collected from electronic records; which, summarised clinic letters from both the consultant and diabetic nurse team. Date of diagnosis and initiation of SAP following that was dictated. The number of hypoglycaemic episodes causing admission to hospital and HBA1c was compared before and after SAP was started.

Results There were equal number of males and females. The average age of Type I DM diagnosis was 6 years 2 months. The average age when SAP was commenced was 8 years 1 month.

Average HBA1c prior to SAP was 63.1 and showed 5.3\% improvement following SAP with 59.8. 21.4\% of children showed deterioration in HBA1c. These were males and belonged to an older age group ( $>5$ years). $14.3 \%$ of children did not show any change in their HBA1c.

$29 \%$ of children had a severe hypoglycaemic episode prior to SAP. Following SAP, 7.1\% of children had a severe hypoglycaemic episode.

Conclusion NICE 2016 has recommended the use of SAP in improving the quality of life for people with Type I DM. The audit highlighted improvement in glucose control with positive effect on HBA1c and reduction in hypoglycaemic episodes. This can be explained with increased adherence to treatment with the use of automatic insulin monitoring and delivery. Ultimately, NHS resource and cost saving is achieved; with the quicker target blood glucose and reduced hospital admissions related to Type I DM disease complications.

\section{G238(P) ABSTRACT WITHDRAWN}

\section{G239(P) A REVIEW OF CHILDREN PRESENTING WITH A NEW DIAGNOSIS OF DIABETES}

CS Cockburn, B Rayen. Paediatic Services, Dumfries and Galloway Royal Infirmary, Dumfries, UK

\subsection{6/archdischild-2018-rcpch.232}

Introduction Diabetic ketoacidosis (DKA) can be a life threatening presentation of Type 1 diabetes in children and young people. Data had previously been collected (from January 2007 - August 2009) that demonstrated that 30\% of newly diagnosed diabetics presented in DKA. Following these results and using the Parma study, a public health awareness campaign was completed using $(\mathrm{P}) \mathrm{s}$ designed by local school children to try and improve public awareness around presentations of diabetes.

Aims To review whether children presented in DKA as their first diagnosis of Type 1 diabetes and whether they had presented to health services on more than one occasion prior to diagnosis.

Methods A list of patients was collected from the Diabetic Team caseload and SCI diabetes database to identify children and young people diagnosed between January 2014 and July 2017. Case notes were retrospectively reviewed using a proforma that was developed in consultation with the diabetes team. 33 patients were included.

Results The majority of patients continued to be diagnosed by GP $(58 \%)$, while out of hours $(\mathrm{OOH})$ made $18 \%$ of diagnoses, $12 \%$ were made by the emergency department (ED) and $12 \%$ by paediatrics. Previously $55 \%$ of diagnoses were made by the GP, $10 \%$ by ED and $20 \%$ by paediatrics. Six of the thirty three patients presented in DKA (19\%) with the remaining $81 \%(27 / 33)$ presenting 'walking wounded'. In comparison to the previous data collection which had demonstrated $30 \%$ (6 of 20) presented with DKA. 42\% of patients were diagnosed at their first presentation, with a further $21 \%$ on their second presentation, $9 \%$ presented on three or more occasions before they were diagnosed.

Conclusion There has been a reduction in percentage of new diabetic children presenting in DKA to services. This may suggest a greater awareness in the community and could be related to a previous public health campaign. It is still concerning that almost 20\% (1 in 5) new diabetics present with DKA and that only $42 \%$ of patients were diagnosed at initial presentation. On some occasions diabetes was suspected but the patients were asked to return with a urine sample before referral to paediatric services was made. 


\section{Paediatricians with Expertise in Cardiology Special Interest Group}

\section{G240 USING THE DELPHI METHOD TO DEVELOP CONSENSUS ON A REFERRAL PATHWAY FOR POTENTIALLY ABNORMAL NEURODEVELOPMENT IN CHILDREN WITH HEART DISEASE}

${ }^{1}$ A Hoskote, ${ }^{2} \mathrm{M}$ Lakhanpaul, ${ }^{1} \mathrm{~V}$ Banks, ${ }^{1} \mathrm{~J}$ Wray, ${ }^{1} \mathrm{~K}$ Brown. ${ }^{1}$ Critical Care and Cardiorespiratory Division, Great Ormond Street Hospital for Children NHS Foundation Trust, London, UK; ${ }^{2}$ Community Child Health, UCL Great Ormond Street Institute of Child Health, London, UK

\subsection{6/archdischild-2018-rcpch.233}

Introduction Children with congenital heart disease (CHD) have complex medical and neurodevelopmental needs. Our aim was to develop a professional consensus-based protocol for referral pathways following the application of the Brief Developmental Assessment (BDA) - an early recognition tool which allows categorisation of a child's current neurodevelopmental status as GREEN (appropriate for age), RED (delayed) or AMBER (equivocal). The BDA has been validated in age groups covering 4 months to 5 years, and supports specialist referral by providing targeted additional and helpful information on the child's development.

Methods The Delphi consensus process involved emailed survey questionnaires, eliciting individual decisions, aggregating group responses and providing formal feedback of group choices. A survey detailing two scenarios - a child identified with red $B D A$ and a child identified with amber $B D A$ - was sent to 80 key professionals and stakeholders involved in managing children with CHD seeking agreement on clear referral pathways on identification of red or amber BDA.

Results The survey was completed by 77 Delphi panel members in Round 1 and 73 in Round 2. For red BDA, there was strong agreement that children should be under the care of a community paediatrician and local child development team, and that referral to a community paediatrician should be undertaken at the point of first assessment. For amber BDA, there was consensus that the child should be under the care of a paediatrician with expertise in cardiology (PEC) (or general paediatrician if no PEC) based at their local hospital, and if not under the care of a PEC that it is the responsibility of the child's paediatric cardiologist to refer them to a PEC.

Conclusions The Delphi process helped facilitate consensus on referral pathways for specialist assessment/treatment following red and amber BDA. On notification of abnormal BDA from the tertiary hospital, the PEC (or general paediatrician if no PEC) at the local hospital and the health visitor are key health professionals who can link a child with CHD and developmental problems with the local child development team for appropriate neurodevelopmental assessment and intervention.

\section{G241 \\ INCIDENCE AND OUTCOMES OF INFANTS WITH CONGENITAL DIAPHRAGMATIC HERNIA ASSOCIATED WITH CARDIOVASCULAR MALFORMATION: A 10 YEARS RETROSPECTIVE STUDY}

${ }^{1} \mathrm{~T}$ Newman, ${ }^{1} \mathrm{M}$ Weidenmuller, ${ }^{1,2}$ Y Singh. ${ }^{1}$ School of Clinical Medicine, University of Cambridge, Cambridge, UK; ${ }^{2}$ Neonatology and Paediatric Cardiology, Cambridge University Hospitals, Cambridge, UK

\subsection{6/archdischild-2018-rcpch.234}

Background Congenital diaphragmatic hernia (CDH) occurs in 1.7-5.7/10,000 live births and is associated with significant neonatal mortality. It is reported to be associated with congenital cardiovascular malformations (CCM) in up to $10.6 \%$ cases, an association which significantly increases mortality.

Aims To study the incidence and outcomes of infants with $\mathrm{CDH}$ associated with significant $\mathrm{CCM}$ in a UK tertiary neonatal referral centre.

Methods PA retrospective observational study involved all infants diagnosed with $\mathrm{CDH}$ and admitted to our maternity hospital between Jan 2007-Dec 2016. Data was collected from electronic patient records.

Results A total of 52 infants diagnosed with CDH were identified. median gestation was 38 weeks (IQR: 36-39 weeks) and average birth weight was 2820 grams (IQR: 2200-3260 grams).

10 infants $(19 \%)$ had associated CCM as shown in table 1. PFO and PDA were excluded as thought normal findings in this population.

Overall survival in our cohort was 79\% (41/52) whilst 11 patients $(21 \%)$ died during infancy. 4 of the 10 infants $(40 \%)$ with $\mathrm{CDH}$ and CCM died whilst mortality was $17 \%$ in infants without such association. Pre-operative mortality was higher in infants with CCM, 30\% Vs 12\% in infants without CCM. Half of the infants with CCM needed transfer to specialist ECMO cardiac centres. Only 2 of the 42 infants without CCM needed transfer for ECMO.

Conclusion In our cohort, there was an increased incidence of significant CCM concomitant with $\mathrm{CDH}$ than previously reported. Moreover, there was lower incidence of mortality in our cohort. Associated CCM, however, still greatly increased the risk of requiring additional specialist care and increased mortality rates as compared to $\mathrm{CDH}$ alone.

Abstract G241 Table 1 Types of congenital cardiac malformation associated with $\mathrm{CDH}$

\begin{tabular}{ll}
\hline Type of congenital cardiac malformation & No of cases (\%) \\
\hline Atriovantricular septal defect (AVSD) & $3(30 \%)$ \\
Ventricular septal defect (VSD) & $2(20 \%)$ \\
Hypoplastic left heart syndrome & $1(10 \%)$ \\
Tetralogy of Fallot (TOF) & $1(10 \%)$ \\
Coarctation of aorta & $1(10 \%)$ \\
Ebstein anomaly & $1(10 \%)$ \\
\hline
\end{tabular}

